\title{
Molecular evolution of a central region containing B cell epitopes in the gene encoding the p67 sporozoite antigen within a field population of Theileria parva
}

\author{
Isaiah Obara • Seitzer Ulrike • Tony Musoke • \\ Paul R. Spooner • Ahmed Jabbar • David Odongo • \\ Stephen Kemp • Joana C. Silva $\cdot$ Richard P. Bishop
}

Received: 28 May 2014 / Accepted: 30 January 2015 / Published online: 13 February 2015

(C) The Author(s) 2015. This article is published with open access at Springerlink.com

\begin{abstract}
Protective immunity induced by the infective sporozoite stage of Theileria parva indicates a potential role for antibodies directed against conserved serologically reactive regions of the major sporozoite surface antigen $p 67$ in vaccination to control the parasite. We have examined the allelic variation and determined the extent of $\mathrm{B}$ cell epitope polymorphism of the gene encoding $p 67$ among field isolates originating from cattle exposed to infected ticks in the Marula area of the rift valley in central Kenya where the African cape buffalo (Syncerus caffer) and cattle co-graze. In the first of two closely juxtaposed epitope sequences in the central region of the p67 protein, an in-frame deletion of a seven-amino acid segment results in a truncation that was observed in parasites derived from cattle that co-grazed with buffalo. In contrast, the variation in the second epitope was primarily due to nonsynonymous substitutions, resulting in relatively low overall amino acid conservation in this segment of the protein. We also observed polymorphism in the region of the protein adjacent to the two defined epitopes, but this was not sufficient to provide statistically significant evidence for positive selection. The data indicates
\end{abstract}

I. Obara $(\bowtie) \cdot$ T. Musoke $\cdot$ P. R. Spooner $\cdot$ D. Odongo $\cdot$ S. Kemp $\cdot$ R. P. Bishop

International Livestock Research Institute (ILRI), P.O. Box 30709, Nairobi 00100, Kenya

e-mail: i.obara@cgiar.org

S. Ulrike · A. Jabbar

Veterinary Infection Biology and Immunology, Research Center Borstel, Parkallee 22, 23845 Borstel, Germany

\section{J. C. Silva}

Institute for Genome Sciences, Department of Microbiology and Immunology, University of Maryland School of Medicine,

Baltimore 21201, MD, USA that B cell epitopes previously identified within the $p 67$ gene are polymorphic within the Marula field isolates. Given the complete sequence identity of the $p 67$ gene in all previously characterized $T$. parva isolates that are transmissible between cattle by ticks, the diversity observed in $p 67$ from the Marula isolates in combination with the clinical reaction of the infected cattle is consistent with them originating from ticks that had acquired T. parva from buffalo.

Keywords B cell epitopes $\cdot$ Theileria parva $\cdot p 67$ gene

\section{Introduction}

Theileria parva is an apicomplexan protozoan parasite of cattle that is transmitted by the tick Rhipicephalus appendiculatus and is the causal agent of East Coast fever, a frequently fatal disease of cattle in eastern and southern Africa (Norval et al. 1992). The tick inoculates T. parva sporozoites that are infective to the mammalian host, and these differentiate rapidly into an intracellular schizont that immortalizes bovine lymphocytes; subsequently, T. parva differentiates into a piroplasm stage that is infective to erythrocytes. Transmission to cattle can originate from ticks that have fed on African Cape buffalo (Syncerus caffer), the major wildlife reservoir of $T$. parva, in which case, there is a different clinical syndrome, known as "corridor disease" involving low levels of schizont parasitosis and piroplasm parasitemia. Transmission by ticks that have previously fed on other cattle results in typical East Coast fever symptoms with higher levels of parasites. Previous studies using both variable number tandem repeats (VNTRs) and antigen genes have revealed genetic differences between parasites 
transmissible among cattle and those found in buffalo (Oura et al. 2011; Pelle et al. 2011), suggesting that the two sets of parasites represent distinct populations.

T. parva has been the subject of more than 40 years of research aimed at development of a recombinant vaccine using antigens derived from both the sporozoite and schizont stages (reviewed in Morrison 2009; McKeever et al. 1999). The closest approach to an effective anti-sporozoite vaccine to date has used a recombinant version of p67, the major sporozoite surface antigen of T. parva. The p67 protein induces a consistent level of $70 \%$ efficacy against either heterologous or homologous needle challenge using sporozoite stabilates in the laboratory (Musoke et al. 1992; Bishop et al. 2003) and reduced severe disease by approximately $45 \%$ in field trials where cattle were exposed to infective ticks (Musoke et al. 2005). Five regions of the p67 protein sequence that are reactive with anti-sporozoite monoclonal antibodies and also correlate with the results of an in vitro parasite-neutralizing assay have been identified using Pepscan analysis (Nene et al. 1999). The central region of the $p 67$ gene includes two closely linked epitopes that are the target of host B cell responses and whose sequences in the $T$. parva Muguga reference stock are ${ }^{169}$ TKEEVPPADLSDQVP ${ }^{183}$ and ${ }^{209}$ LQPG KTS ${ }^{215}$. These are subsequently referred to as epitopes 1 and 2 , respectively.

While the $p 67$ gene is variable among buffalo-derived isolates, the predicted p67 protein appears to be invariant in cattle-derived stocks of T. parva that are transmissible between cattle by ticks (Nene et al. 1996, 1999; Musoke et al. 2005). A method of live vaccination known as infection and treatment (ITM) was developed approximately 40 years ago (Radley et al. 1975), and there is evidence that the protection induced by ITM is attributable primarily to class I major histocompatibility complex (MHC)-restricted $\mathrm{CD} 8^{+}$cytotoxic $\mathrm{T}$ cells (McKeever et al. 1994). However, this cytotoxic T cell response is strain-specific and strongly dependent on the bovine class I MHC phenotype of the host (Taracha et al. 1995). This may constrain development of a broadly cross-protective recombinant vaccine that mimics the cellular responses induced by ITM and highlights the potential importance of the conservation of the p67 antigen in cattle-derived T. parva. Extensive sequence divergence in the central region of the p67 gene in buffalo-derived parasites from South Africa has recently been described (Sibeko et al. 2010). However, the p67 vaccine has not yet been tested in areas where the parasite challenge is mainly from $T$. parva originating from buffalo. We focus in the study described herein on in-depth analysis of p67 B cell epitope polymorphism in cattle-infective isolates from a specific geographical locality in central Kenya where buffalo and cattle co-graze. In addition, we examine whether any codons show signatures of positive selection in the central region of the $p 67$ gene.

\section{Materials and methods}

Parasite isolates and genomic DNA extraction

Genomic DNA preparations were made from 18 cryopreserved pellets of $10^{7}$ T. parva schizont-infected lymphocyte cultures initially isolated from cattle that co-grazed with the African Cape buffalo, using the DNeasy ${ }^{\circledR}$ Tissue Kit (Qiagen, Germany) according to the manufacturer's instructions. The cattle were part of a field trial of ITM vaccines performed in the year 2000 to explore protection afforded to immunized animals that received a $T$. parva challenge from buffalo-associated ticks at Marula farm, central Kenya (Pelle et al. 2011). The trial was carried out in strict accordance with the recommendations in the standard operating procedures of the ILRI's Institutional Animal Care and Use Committee (IACUC). Fifty-three of the 113 tick-exposed cattle developed clinical disease and died; mortality was observed in $40 \mathrm{immu}-$ nized animals and 13 of the control cattle. Most animals exhibited clinical and parasitological features typical of those induced by buffalo-derived T. parva (Norval et al. 1992) with a low schizont parasitosis and low or, in some cases, no piroplasm parasitemia. As in Table 1, among the 18 cattle from which schizont-infected lymphocyte cultures were generated from lymph node biopsies were nonimmunized controls, as well as those immunized with one of the following stabilates: T. parva Marikebuni stabilate 3014 (Morzaria et al. 1995), T. parva Marikebuni stabilate 316 (Payne 1999), and T. parva composite trivalent Muguga cocktail stabilate FAO1 (Morzaria et al. 1999). All cattle were monitored daily from day 17 after exposure, and the clinical reactions are summarized in Table 1.

Amplification and sequencing of the gene encoding T. parva p67

The amplification of the gene encoding p67 was performed using genomic DNA templates with primer pairs IL 6133 (ACAAACACAATCCCAAGTTC) and IL 7922 (CCTTTA CTACGTTGGCG), designed to amplify a 900-base pair (bp) internal fragment containing part of exon 1 and part of exon 2 separated by the 29-bp intron sequence (Nene et al. 1996). These conserved primers amplify multiple $p 67$ alleles (Nene et al. 1996; Sibeko et al. 2010). The polymerase chain reaction (PCR) reagents and cycling parameters were as described previously (Nene et al. 1996). The PCR products were purified after fractionation through $1.2 \%$ agarose gels, using the QIAquick gel extraction kit (Qiagen). The amplicons were sequenced bidirectionally using IL 6133 forward and IL 7922 reverse primers using Sanger dideoxy technology. Sequences were assembled and edited using the CLC Genomics Workbench version 6.0. 
Table 1 Classification of T. parva p67 alleles based on indels and B cell epitope sequence variation

\begin{tabular}{|c|c|c|c|c|c|c|c|c|}
\hline \multirow{2}{*}{$\begin{array}{l}\text { I-parva } \\
\text { strain }\end{array}$} & \multirow{2}{*}{$\begin{array}{l}\text { Animal } \\
\text { No. }\end{array}$} & \multirow{2}{*}{$\begin{array}{l}\text { Vaccine } \\
\text { stabilate }\end{array}$} & \multirow{2}{*}{$\begin{array}{l}\text { p67 sequence } \\
\text { Accession }\end{array}$} & \multicolumn{2}{|c|}{ Antibody epitope } & \multicolumn{2}{|c|}{ Deletion (bp) } & \multirow{2}{*}{$\begin{array}{l}\text { Allele } \\
\text { type }\end{array}$} \\
\hline & & & & Epitope 1 & Epitope 2 & 129 & 174 & \\
\hline Muguga & & & $\mathrm{XM} 758212.1$ & TKEEVPPADLSDQVP & LQPGKIS & + & - & \\
\hline KNP2 & & & AF079177.1 & $\ldots \ldots$ & $\ldots \ldots$ & + & - & 1 \\
\hline Marula & $\mathrm{N} 16(\mathrm{SRD})$ & 316 & LK0 54513 & & & + & - & \\
\hline Marula & $\mathrm{N} 32(\mathrm{FD})]$ & FAO 1 & Dito & (n) & $\cdots$ & + & & \\
\hline Buffalo & & & $\mathrm{U} 40703.1$ & 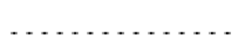 & $\ldots \ldots$ & - & - & \\
\hline Marula & N4 (NR) & FAO 1 & LK0 54504 & $\ldots \ldots \ldots$ & P..... & - & - & \\
\hline Marula & N17 (MR) & FAO 1 & LK054505 & $\ldots \ldots . .6$. & $\ldots .$. & - & - & 2 \\
\hline Marula & N25 (NR) & 316 & LK054506 & & & - & _- & \\
\hline Marula & N3 (SR) & FAO 1 & & (n) & $\cdots$ & & & \\
\hline Marula & N31 (NR) & FAO 1 & LK0 54507 & $\ldots \ldots \ldots$ & P...... & - & - & \\
\hline KNPWB_35 & & & JX 442249.1 & ........------- & KN . R . D & - & + & \\
\hline We124_2-5 & & & $J X 442248.1$ & . . . . . . - ------ & $-\mathrm{KN}, \mathrm{R} \cdot \mathrm{D}$ & - & + & \\
\hline Marula & N7 (MR) & 3014 & LK054508 & ........ -------- & $\mathrm{KN}, \mathrm{R}, \mathrm{D}$ & - & + & \\
\hline Marula & N8 (SRD) & 316 & & & & & & \\
\hline Marula & N1 (NR) & 3014 & LR054509 & 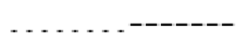 & KN . R.D & - & + & \\
\hline Marula & N19 (MR) & Control & & & & & & \\
\hline Marula & N2 7 (NR) & 316 & & & & & & \\
\hline Marula & N10 (NR) & Control & & & & & & 3 \\
\hline Marula & $\mathrm{N} 12(\mathrm{MR})$ & Control & IRO 054510 & $\ldots . . . . .------$ & $\mathrm{KN}, \mathrm{R}, \mathrm{D}$ & - & + & \\
\hline Marula & $\mathrm{N} 26(\mathrm{NR})$ & Control & Lex $000+0$ & ......... & $-K N N_{-}-D$ & & & \\
\hline Marula & $\mathrm{N} 2 \mathrm{~B}$ (NR) & FAO 1 & & & & & & \\
\hline Marula & N11 (NR) & 316 & LK054511 & . . . . . . - ------ & KN,$R . D$ & - & + & \\
\hline Marula & N33 (SRD) & FAO 1 & LK054512 & ......... -------- & $-K N$. R . D & - & + & \\
\hline Ita $8-13$ & & & $\mathrm{JX} 442245.1$ & $\ldots . . . I \ldots-------$ & $\mathrm{KN}, \mathrm{B}, \mathrm{D}$ & - & + & \\
\hline Mar1_49 & & & $J X 442244.1$ & $\ldots . . . I \ldots-------$ & $-\mathrm{KN}, \mathrm{R}, \mathrm{D}$ & - & + & \\
\hline HIP5_1-16 & & & $\operatorname{TX} 442246.1$ & $\ldots I \ldots K-1 .--n$ & $-\mathrm{KN}, \mathrm{R}, \mathrm{D}$ & - & + & \\
\hline KNP102-9 & & & $\mathrm{JX} 442247.1$ & $\ldots . . . I \ldots------$ & KN . R . D & - & + & \\
\hline
\end{tabular}

Clinical reactions of cattle exposed to tick challenge at Marula farm are in parenthesis and abbreviated as follows: $S R D$ severe reaction and died, $S R$ severe reaction, $M R$ mild reaction, $N R$ nonreactor, $F D$ found dead. Curly braces enclose animals from which the same sequence type was obtained. "." Epitope positions which have a conserved residue; “-” gapped positions in the antibody epitopes

p67 linear epitope sequence alignments, variant calling, and sequence logo creation

Basic Local Alignment Search Tool (BLAST) version 2.2.29+ (Altschul et al. 1990) search against a local database containing a repertoire of T. parva isolates in East and South Africa assigned the Marula query nucleotide sequences based on alignment scores to one of the four previously identified T. parva p 67 allele categories. The 13 sequences derived from T. parva 067 antigen gene included in the local database were retrieved using the BLAST utility of the NCBI nucleotide database and had GenBank accessions: XM 758212.1, U40703.1, AF079177.1, JX442251.1, JX442249.1, JX442247.1，JX442246.1，JX442245.1，AF079176.1, JX442248.1, JX442244.1, JX442250.1, and AF079175.1. The sequences included in the local database were used as references in a multiple sequence alignment with $p 67$ sequences from isolates genotyped in this study covering the polymorphic central region of the gene. The alignment was generated with MAFFT version 7.122 using the L-INS-i option (Katoh et al. 2002), with minor subsequent manual adjustments. The program Seq2Logo version 1.2 (Thomsen and Nielsen 2012) was used to create a Kullback-Leibler sequence logo by calculating the information content as a function of the observed and background probability of residues at each position in the alignment region spanning the mapped targets of host B cell responses. In addition, estimates of $p 67$ gene polymorphism, $\pi$, calculated as the average number of nucleotide differences per site, were generated with DnaSP version 5 (Librado and Rozas 2009).

Selection of best-fit nucleotide substitution models for T. parva $\mathrm{p} 67$ evolution

The phylogeny of the T. parva p67 antigen gene sequences was inferred from an optimal subset of the alignment generated as described above. This alignment subset was determined by the number of residues in gap-free columns by the program MaxAlign version 1.1 (Gouveia-Oliveira et al. 2007). We performed a preliminary reconstruction of the phylogenetic relationships between sequences using a neighbor-joining (NJ) tree (Saitou and Nei 1987) under the Jukes and Cantor (JC) 
model. The NJ-JC tree served as the basis for calculating the likelihood scores for 56 different nucleotide substitution models with varying sets of substitution rate parameters capable of accommodating rate heterogeneity between sites. The best fitting model for the $p 67$ data was selected based on the Akaike information criterion ( $\mathrm{AIC}=-2 \operatorname{lnL}+2 K$ ), a function of the maximized $\log$-likelihood $(\mathrm{lnL})$, and the number of estimated parameters $(K)$ for a model (Wagenmakers and Farrell 2004), using the program Modeltest version 3.06 (Posada and Crandall 1998).

\section{Assessing model fits by using AIC}

The phylogeny of the T. parva p67 antigen gene sequences was estimated with the maximum likelihood (ML) criterion and the parameters from the best-fit nucleotide substitution model, using PAUP version 4 (Swofford 2002). Estimations of $d_{\mathrm{S}}$ and $d_{\mathrm{N}}$ were performed using the codeml program from the PAML package version 4 (Yang 2007). These estimates were obtained under two different models of evolution: the nearly neutral (M1) and the positive selection (M2) models. M1 assumes two categories of sites, one evolving under purifying selection $\left(0<d_{\mathrm{N}} / d_{\mathrm{S}}<1\right)$ and the other neutral $\left(d_{\mathrm{N}} / d_{\mathrm{S}}=1\right)$. Model M2 adds to these an additional category of positively selected sites $\left(1<d_{\mathrm{N}} / d_{\mathrm{S}}\right)$. Both models were fitted by allowing different nucleotide frequencies for each codon position. The AIC was used to seek the fitted model where the information loss based on the expected relative Kullback-Leibler distance as defined by AIC differences $\left(\Delta_{\mathrm{i}}\right)$ was minimal.

\section{Results}

p67 gene variants among T. parva isolates from Marula ranch (central Kenya)

We sequenced the central region of the T. parva 067 gene from a collection of cultured schizont-infected lymphocytes recently isolated from cattle which had been exposed to tick challenge within Marula farm where the African buffalo co-grazed (Pelle et al. 2011). We generated an alignment of the amplified internal fragment of the T. parva 067 gene, which contains $900 \mathrm{bp}$ in the reference T. parva Muguga isolate. The alignment contains 27 sequences, nine of which are available in GenBank and 18 of which are derived from the Marula isolates (Fig. 1). There were ten different p67 sequences represented among the total of 18 from Marula, each of which was derived from a schizont culture isolate from a different animal (Table 1). None of the ten sequences was the same as the p67 sequences of the $T$. parva stocks within the immunizing stabilates, all of which are identical to one another (Nene et al. 1996, M. Norling and J. Silva in preparation). The Marula sequences described in this report are available from the European Nucleotide Archive at http://www.ebi.ac.uk/ ena/data/view/LK054504-LK054513, and animals from which the same sequence type was obtained are shown in Table 1. Among the alleles sequenced from the Marula isolates, the overall DNA polymorphism, $\pi$, within and outside the mapped B cell epitopes in the amplified central fragment of the $p 67$ gene was 0.19627 (19.6\%). All sequences were divided into three allele types, 1 through 3 , defined by the presence or absence of two indels (Table 1). The indel set comprised both a 129-bp deletion (allele type 1) and an additional 174-bp deletion (allele type 3). Fifty-seven percent of the sequences were within $p 67$ allele type 3 based on the indels and only $10 \%$ were within allele size type 1 . Approximately one third of all sequences in the Marula isolate collection were assigned to $p 67$ allele type 2 on the basis of sequence similarity to allele type 1 combined with the absence of the 129-bp deletion (Table 1).

Sequence polymorphism between antibody epitopes within the $p 67$ gene for Marula isolates

We determined the degree of sequence variation within epitopes 1 and 2 of the T. parva p67 gene in the Marula field isolates. The predicted protein sequences were compared with the previously characterized p67 protein sequences derived from East and South African isolates (Sibeko et al. 2010). Within the region encoding the mapped epitopes, the high level of nucleotide sequence polymorphism translated into coding changes that resulted in amino acid mutations relative to the T. parva Muguga reference allele (XM_758212.1), a cattle-type $p 67$ sequence (Nene et al. 1996). These variants are shown in Table 1. Among the sequences assigned to allele types 1 and 2, epitope 1 was remarkably conserved, perfectly matching the reference sequence. However, an in-frame deletion of a seven-amino acid segment was observed in a majority of the Marula sequences, all of which were assigned to allele type 3 . This truncated epitope sequence resulting from the deletion appears to be a unique feature of the $p 67$ indeldefined allele type 3 . In contrast, epitope 2 sequences were less homogeneous among the sequences assigned to allele types 1 and 2 due to a nucleotide substitution that conferred a predicted amino acid change, with the consensus glutamine, ${ }^{210} \mathrm{Q}$, being replaced by proline, $\mathrm{P}$. Additionally, there were nonsynonymous mutations in epitope 2 relative to the reference sequence in all of the isolates assigned to $p 67$ allele type 3.

Position-specific enrichment and depletion of residues within the p67 linear epitopes

Information content, a function of the observed and background probability for a particular column in the multiple sequence alignment, was calculated for each position spanning the mapped epitopes to assess their relative contributions 


\begin{tabular}{|c|c|c|c|c|c|c|c|}
\hline $8212-1$ & IPTPUSEEIITPTLQAQ & TKEEVPRADLSDQVA & SNG & SDSEEEDNKSTSSKDEKE & LKKIf & & QDLNSKQQQTGUSDI \\
\hline $079177-1$ & $A \ldots \ldots \ldots \ldots$ & $\ldots \ldots \ldots \ldots$ & $\ldots$ & $\ldots \ldots$. . ED.SIGI . RG & & P........ & $\ldots \mathrm{KT} \ldots \mathrm{KN} \ldots \mathrm{GP} . \mathrm{G}$ \\
\hline $1513(2)$ & $\ldots \ldots \ldots \ldots \ldots$ & $\ldots \ldots \ldots \ldots \ldots$ & $\ldots$ & $\ldots \ldots \ldots \ldots \ldots$ & $\cdots$ & $\ldots \ldots$. & \\
\hline $703-1$ & $\ldots \ldots \ldots \ldots$ & $\cdots \cdots$ & - & $\ldots \ldots \ldots \ldots \ldots$ & . & $\cdots$ & $\ldots \ldots$ \\
\hline K054504 & $\ldots \ldots \ldots \mathrm{T}$. & $\ldots \ldots \ldots$ & .. & ......GD. SLGT . RN & & p..... & $\ldots k \ldots \ldots$. \\
\hline 2054505 & $\ldots \ldots \ldots \ldots$ & & & $\ldots \ldots \ldots \ldots \ldots$ & & & \\
\hline $2054506(2)$ & $\cdots \cdots \cdots \cdots$ & $\ldots$ & $\cdots$ & $\ldots \ldots \ldots \ldots$ & & $\ldots \ldots$ & $\cdots$ \\
\hline $\operatorname{LK054507}$ & P............ & $\ldots \ldots \ldots$ & $\cdots$ & .......GD.SLGT . RN & & $P \ldots \ldots D$ & $\ldots$ K............. \\
\hline$J X 442249-1$ & $\ldots \ldots \ldots \ldots \ldots$ & & $-s s$ & . . Q..S-------. EN & GNDG & KN.R DGKNGAA. & ------------------ \\
\hline$J X 442248-1$ & $\ldots \ldots \ldots \ldots \ldots$ & .. & $-s s$ & ...Q. Q.S-------. $\mathrm{BN}$ & GNGG. & KN.R.DGKNGP & \\
\hline LK054508 & $\ldots \ldots \ldots \ldots \ldots$ & & $-s s$ & $\ldots$. Q . S-S----- . EN & GDNT & KN.R.DGKNGD & \\
\hline $\operatorname{Ro5} 4509(4)$ & $\ldots \ldots \ldots . .$. & & $-s s$ & ...Q. Q.S----- . EN & GGDG. & KN. R. DGKNGDA. & \\
\hline $\operatorname{LK054510(4)}$ & & .. & $-s s$ & $\ldots$ Q Q . S------ . EN & GDNV. & KN . R D DGNGDA. & \\
\hline LK054511 & $\ldots \ldots \ldots \ldots \ldots$ & & $-s s$ & ...Q. Q.S------. $\mathrm{EN}$ & GDNV & KN. R DGKNGDA. & ------ \\
\hline $\operatorname{LK054512}$ & $\ldots \ldots$. K...... & $\ldots \ldots \ldots$ & $-s s$ & $\ldots Q Q . . S------. \Xi N$ & GGDG. & KN. R.DGKNGDA. & - - - - - - - - - - - - - - - \\
\hline$J X 442245-1$ & $\ldots \ldots \ldots$. . HT. & ....I. . K- - - & $-S D$ & $. \Xi \ldots Q . . S------. \Xi N$ & EDDV & KN . R. DRKNGAA. & - - - - - - - - - - - - - - - \\
\hline$J \times 442244-1$ & $\ldots \ldots$...... & ....I. I K- - - - & $-S D$ & .E. .Q..S------- . EN & EDDV. & IN . R.DRKNGAA. & 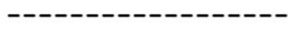 \\
\hline $4442246-1$ & $\ldots \ldots \ldots$. . . HT. & $\ldots . . . \mathrm{I}-\cdots$ & $-S D$ & .E. . Q. S-S----- . EN & EDDV & KN R DRKNGAA. & --- \\
\hline $442247-1$ & $\ldots \ldots$....... . HT. & ....I. I K - - - - - & $-S D$ & $. \Xi \ldots Q . S------. E N$ & & KN . R . DRKNG A. & \\
\hline
\end{tabular}

Fig. 1 Multiple deduced amino acid sequence alignment of a section of the amplified internal fragment of the T. parva p67 gene generated using the multiple sequence alignment program, MAFFT version 7.122 (Katoh et al. 2002) "." Amino acid (aa) positions which have a conserved residue; “-” gapped aa positions; number of Marula isolates carrying an allele is indicated in parentheses. The epitopes recognized by antisporozoite monoclonal antibodies are boxed to epitope polymorphisms and visualized in a KullbackLeibler logo. As can be seen in Fig. 2, Seq2Logo (Thomsen and Nielsen 2012) captures the extent of B cell epitope polymorphism in $T$. parva p67 among field isolates by taking advantage of pseudo count estimates and sequence weighting to deal with data redundancy and the low number of observations, respectively. The amino acids enriched at each epitope position are shown on the positive $y$-axis, and the corresponding depleted amino acids are on the negative $y$-axis.
Analysis of Marula $p 67$ sequences revealed an excess of nucleotide substitutions resulting from transitions relative to transversions

To investigate the nature of diversity in the sequenced $p 67$ alleles, the model of nucleotide substitution that represents the best fit to the data, to be used for phylogenetic inference, was established by statistical testing. This was assessed by the AIC, a function of the maximized $\log$-likelihood $(\ln L)$, and the
Fig. 2 Kullback-Leibler logo for sequences corresponding to B cell epitopes 1 and 2 for sporozoite-neutralizing antibodies on the T. parva $\mathrm{p} 67$ based on a multiple alignment of the predicted amino acid sequences within the central region. Enriched amino acids (aa) are shown on the positive $y$-axis and depleted (underrepresented) amino acids on the negative $y$-axis. The height of the column of aa residues represents the level of conservation at a particular position, while the relative residue height denotes its frequency. Polar aa is depicted in green, basic aa in blue, acidic aa in red, and hydrophobic aa in black

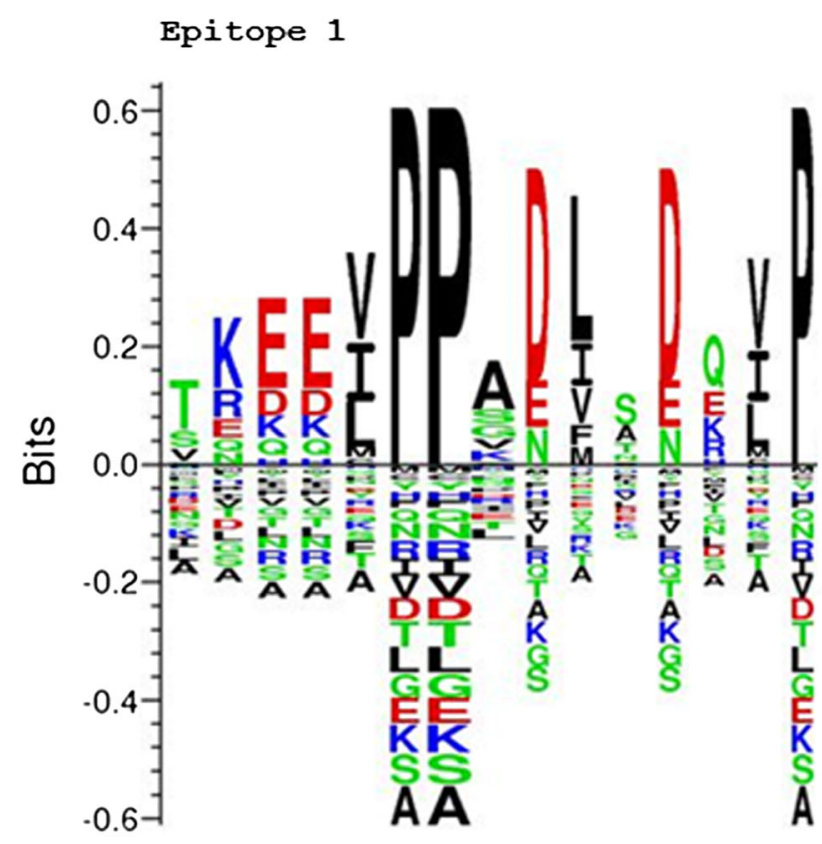

Epitope 1

Epitope 2

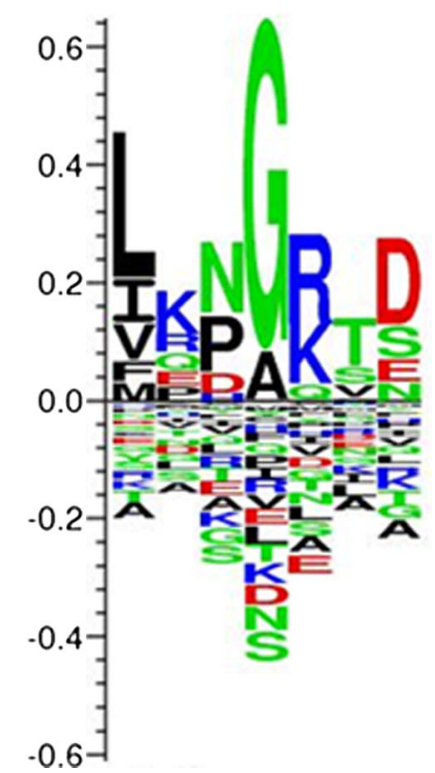


number of estimated parameters $(K)$ for a model. The $p 67$ dataset was best explained by the TIM $+\mathrm{I}+\mathrm{G}$ model of evolution. This model assumes variable base frequencies, variable transition rates, two transversion rates, a proportion of invariable sites (I), and a gamma distribution with shape parameter $(\alpha)$. With the corresponding base frequencies taken into consideration, maximum likelihood estimates of substitution rates revealed more transitions than transversions. In particular, the A-to-G transition occurred most frequently; $65 \%$ of the sites were invariable $(p-i n v)$, and the gamma shape parameter $(\alpha)$ was less than 1 , the latter indicating a relatively slow rate of evolution at the $p 67$ locus (Table 2).

Spatial clustering of positively selected codons in the $p 67$ gene

We made maximum likelihood inferences of positive selection based on the AIC approach to compare the fit of models M1, the quasi-neutral model, which allows for two site classes $\left(d_{\mathrm{N}} /\right.$ $\left.d_{\mathrm{S}}=1,0<d_{\mathrm{N}} / d_{\mathrm{S}}<1\right)$ relative to a positive selection model, $\mathrm{M} 2$, with three site classes $\left(d_{\mathrm{N}} / d_{\mathrm{S}}=1,0<d_{\mathrm{N}} / d_{\mathrm{S}}<1\right.$, and $\left.d_{\mathrm{N}} / d_{\mathrm{S}}>1\right)$. As shown in Table 3, fitting the codon substitution model that captures the proportion of codons for which $d_{\mathrm{N}} / d_{\mathrm{S}}>1$ within
Table 3 Parameters and Akaike information criterion (AIC) scores of the $p 67$ gene under codon site models

\begin{tabular}{lccccccc}
\hline Model & $K$ & $\operatorname{lnL}$ & $\mathrm{AIC}_{\mathrm{c}}$ & $\Delta_{i}$ & $d_{\mathrm{N}} / d_{\mathrm{S}}<1$ & $d_{\mathrm{N}} / d_{\mathrm{S}}=1$ & $1<d_{\mathrm{N}} / d_{\mathrm{S}}$ \\
\hline M1 & 49 & 1260 & 2618 & 2.0 & $p 0.54325$ & $p 0.45675$ & \\
& & & & & $\omega 0.09055$ & $\omega 1.00000$ & \\
M2 & 51 & 1257 & 2616 & 0.0 & $p 0.53538$ & $p 35855$ & $p 0.10607$ \\
& & & & & $\omega 0.11037$ & $\omega 1.00000$ & $\omega 2.95491$
\end{tabular}

The Akaike information criterion (AIC), a function of the maximized $\log$-likelihood $(\operatorname{lnL})$, and the number of estimated parameters $(K)$ used to select the best fitting model. $\omega$ is the Akaike weight and $p$ gives the proportion of codon sites belonging to each of the $d_{\mathrm{N}} / d_{\mathrm{S}}$ ratio classes, and $\Delta_{\mathrm{i}}$ denotes AIC differences

the $p 67$ gene provided a better fit than M1 (minimum AIC value). In the best fit model (M2), about $53 \%$ of the codons are strongly conserved (average $d_{\mathrm{N}} / d_{\mathrm{S}}=0.11037$ ), $35 \%$ are consistent with neutral evolution $\left(d_{\mathrm{N}} / d_{\mathrm{S}}=1.0\right)$, and the other $10 \%$ of codons exhibit $d_{\mathrm{N}} / d_{\mathrm{S}}$ values suggesting evolution under positive selection (average $d_{\mathrm{N}} / d_{\mathrm{S}}=2.95491$ ). Bayesian posterior probabilities calculated using Bayes empirical Bayes (BEB) (Yang et al. 2005) in M2 revealed the codons with signatures of positive selection (Table 4). However, in the case of most codons, the standard error interval includes $d_{\mathrm{N}} / d_{\mathrm{S}}=1$,

Table 2 AIC model selection and maximum likelihood estimates from best-fit model (TIM $+\mathrm{I}+\mathrm{G})$

\begin{tabular}{|c|c|c|c|c|c|c|}
\hline Model & $\ln L$ & $K$ & AIC & Delta & Weight & Cum weight \\
\hline $\mathrm{TIM}+\mathrm{I}+\mathrm{G}$ & 3243.4773 & 8 & 6502.9546 & 0.0000 & 0.2086 & 0.2086 \\
\hline $\operatorname{TrN}+\mathrm{I}+\mathrm{G}$ & 3244.5530 & 7 & 6503.1060 & 0.1514 & 0.1934 & 0.4021 \\
\hline $\mathrm{K} 81 \mathrm{uf}+\mathrm{I}+\mathrm{G}$ & 3245.0901 & 7 & 6504.1802 & 1.2256 & 0.1130 & 0.5151 \\
\hline $\mathrm{HKY}+\mathrm{I}+\mathrm{G}$ & 3246.1763 & 6 & 6504.3525 & 1.3979 & 0.1037 & 0.6188 \\
\hline $\mathrm{TIM}+\mathrm{G}$ & 3245.1899 & 7 & 6504.3799 & 1.4253 & 0.1023 & 0.7211 \\
\hline $\mathrm{TrN}+\mathrm{G}$ & 3246.3713 & 6 & 6504.7427 & 1.7881 & 0.0853 & 0.8066 \\
\hline $\mathrm{K} 81 \mathrm{uf}+\mathrm{G}$ & 3246.7419 & 6 & 6505.4839 & 2.5293 & 0.0589 & 0.8645 \\
\hline $\mathrm{HKY}+\mathrm{G}$ & 3247.9426 & 5 & 6505.8853 & 2.9307 & 0.0482 & 0.9136 \\
\hline TIM & 3313.7852 & 6 & 6639.5703 & 136.6157 & $4.50 \mathrm{e}-31$ & 1.0000 \\
\hline K80 & 3358.1204 & 1 & 6718.2407 & 215.2861 & $0.00 \mathrm{e}+00$ & 1.0000 \\
\hline $\mathrm{JC}$ & 3368.9949 & 0 & 6737.9897 & 235.0352 & $0.00 \mathrm{e}+00$ & 1.0000 \\
\hline
\end{tabular}

Maximum likelihood estimate from best-fit model (TIM $+\mathrm{I}+\mathrm{G})$

$\begin{array}{ll}\text { Base frequencies } & \text { Among-site rate variation } \\ \text { freq } \mathrm{A}=0.3548 & \text { Proportion of invariable sites }(\mathrm{I})=0.3332 \\ \text { freqC }=0.2131 & \text { Variable sites }(\mathrm{G}) \\ \text { freqG }=0.2104 & \text { Gamma distribution shape parameter }=06479\end{array}$

freqT $=0.2216$

Substitution model

Rate matrix
$\mathrm{R}$ (a) $[\mathrm{A}-\mathrm{C}]=1.0000$
$\mathrm{R}(\mathrm{b})[\mathrm{A}-\mathrm{G}]=2.5858$
$\mathrm{R}(\mathrm{c})[\mathrm{A}=\mathrm{T}]=1.3319$
$\mathrm{R}(\mathrm{d})[\mathrm{C}-\mathrm{G}]=1.3319$
$\mathrm{R}(\mathrm{e})[\mathrm{C}-\mathrm{T}]=1.7247$
$R$ (f) $[\mathrm{G}-\mathrm{T}]=1.0000$

The Akaike information criterion (AIC), a function of the maximized log-likelihood $(\operatorname{lnL})$, and the number of estimated parameters $(K)$ used to select the best fitting model. Delta denotes the AIC differences. Also shown are the maximum likelihood estimates of base frequencies, nucleotide substitution rates, and rate heterogeneity parameters (proportion of invariable sites and shape parameter of the gamma distribution) 
Table 4 Posterior probabilities (Pr) and estimated $d_{\mathrm{N}} / d_{\mathrm{S}}$ ratios (post mean $\pm \mathrm{SE}$ for $d_{\mathrm{N}} / d_{\mathrm{S}}$ ) calculated using Bayes empirical Bayes (BEB) analysis for sites likely to be under positive selection on the $T$. parva p67 gene identified using the reference stock T. parva Muguga

\begin{tabular}{llll}
\hline $\begin{array}{l}\text { Positively } \\
\text { selected site }\end{array}$ & Amino acid & $\operatorname{Pr}\left(d_{\mathrm{N}} / d_{\mathrm{S}}>1\right)$ & $\begin{array}{l}\text { Post mean } \pm \mathrm{SE} \\
\text { for } d_{\mathrm{N}} / d_{\mathrm{S}}\end{array}$ \\
\hline 151 & $\mathrm{~T}$ & 0.645 & $2.257 \pm 1.363$ \\
186 & $\mathrm{G}$ & 0.868 & $2.815 \pm 1.394$ \\
194 & $\mathrm{~N}$ & 0.686 & $2.259 \pm 1.212$ \\
204 & $\mathrm{E}$ & 0.532 & $1.893 \pm 1.094$ \\
205 & $\mathrm{~L}$ & 0.545 & $1.907 \pm 1.080$ \\
208 & $\mathrm{~T}$ & 0.772 & $2.505 \pm 1.294$ \\
237 & $\mathrm{~L}$ & 0.801 & $2.596 \pm 1.323$ \\
262 & $\mathrm{G}$ & 0.535 & $1.871 \pm 1.047$ \\
277 & $\mathrm{H}$ & 0.745 & $2.469 \pm 1.335$ \\
278 & $\mathrm{Q}$ & 0.671 & $2.247 \pm 1.243$ \\
280 & $\mathrm{~V}$ & 0.654 & $2.238 \pm 1.291$ \\
\hline
\end{tabular}

and the posterior probability of any codon evolving with $d_{\mathrm{N}} /$ $d_{\mathrm{S}}>1$ is never equal to, or larger than, $95 \%$ and therefore not significant at this level.

\section{Discussion}

We present evidence for $p 67$ alleles grouped into three distinct indel types within a single $T$. parva population infecting cattle that were part of a field trial of ITM vaccines designed to evaluate protection afforded to immunized animals that received a $T$. parva challenge from buffalo-associated ticks (Pelle et al. 2011). All of these $\mathrm{p} 67$ alleles were derived from T. parva genotypes, present in the Marula tick population, since although ITM immunization typically induces a persistent carrier state in cattle, both Marikebuni and the Muguga, Serengeti and Kiambu V stocks within the trivalent FAO1 Muguga cocktail, have an identical p67 sequence that differs from the p67 sequences of any of the Marula isolates. Comparable levels of heterogeneity at the T. parva p67 locus have recently been reported in South Africa (Sibeko et al. 2010). Classification relative to previously described alleles was based on presence or absence of 129- and 174-bp indels in the central region of the $p 67$ gene. We show that parasites with a 174-bp deletion (allele type 3 ) were most frequent in the isolates from the cattle under challenge at Marula farm. Whether there is any underlying selective advantage that the deletion might confer is unclear. One possibility is that epitope 1 is truncated and therefore not recognized in parasites containing the 129-bp sequence but with the $174 \mathrm{bp}$ deleted.

The ten different $T$. parva $p 67$ sequences described herein (derived from a total of 18 sequences) were distinct at a minimum of 144 nucleotides excluding sequence reads covering indels. This contrasts strongly with the complete conservation of nucleotides at the $p 67$ locus, including introns and the third base in codons, in cattle-transmissible parasites (Nene et al. 1996, Musoke et al. 2005). We assessed the nature and extent of variation in the two closely juxtaposed B cell epitopes that have been mapped to the central polymorphic region of the p67 protein (Nene et al. 1999). Our analysis demonstrated that all allele type 1 sequences containing the 174-bp sequence but lacking the 129-bp sequence were identical to the prototype Muguga (XM_758212.1) at both epitopes analyzed. This was also the case for the isolates within the indel-defined allele type 2 with the exception of a single variation present in two isolates. The high level of conservation of these epitopes suggests that they are candidates for inclusion in multivalent vaccines with the potential to reduce establishment of infection when challenge occurs with multiple T. parva genotypes. Indeed, neutralizing antibodies directed against conserved epitopes have been found to be correlates of protection against infective sporozoites in several species of parasitic protozoans within the phylum apicomplexa including Plasmodium (Weedall et al. 2007), Eimeria (Wallach et al. 2008), and T. parva (Musoke et al. 1992).

Although studies with monoclonal antibodies have defined 169 TKEEVPPADLSDQVP ${ }^{183}$ as a p67 epitope in T. parva isolates that are transmissible between cattle by ticks (Nene et al. 1999), our analysis reveals a deletion variant in this region that is common to all type 3 alleles, suggesting that it may not represent a B cell epitope in many buffalo-derived T. parva parasites. Interestingly, there is a high level of conservation of the epitope sequence flanking the deletion in the isolates carrying allele type 3 . The amino acid sequence of epitope 2 in allele 3 isolates is also conserved but distinct from that of allele types 1 and 2, although not altered in length. Collectively, these findings support the hypothesis that these p67 regions are potential targets for inclusion in a combinatorial vaccine formulation that includes immunodominant $\mathrm{B}$ cell epitopes. Vaccine strategies similar to those being pursued for the polymorphic merozoite surface protein (MSP) 1 malaria protein could also be applicable to T. parva. In the case of MSP 1, it has been suggested that focusing on both the conserved C-terminal region (Blackman et al. 1994) in combination with polymorphic subtype-restricted epitopes (Tetteh et al. 2005) to create chimeric constructs could be a viable vaccine development strategy. For T. parva p67, a vaccine could also incorporate conserved epitopes located outside the p67 central region, for example, in the C-terminal region in which no polymorphism has been reported, within a single chimeric construct.

We used the multiple sequence alignment generated from the $p 67$ dataset described in Table 1, to investigate what modality of selection may have contributed to the evolution of the $p 67$ gene. Such tests typically assume a particular phylogeny and can be influenced by the nucleotide substitution model 
selected (Yang et al. 1994). After optimization of the parameters, an AIC analysis indicated that the $\mathrm{TIM}+\mathrm{I}+\mathrm{G}$ model of substitution represented the best fit to the $p 67$ polymorphism data. AIC is an efficient alternative to likelihood ratio tests (Posada and Buckley 2004) and has recently been implemented in the identification of the codon substitution model that best fits sequence polymorphisms in a region of VAR2CSAa Plasmodium falciparum protein involved in placental sequestration in the mammalian host (Dahlbäck et al. 2006). We detected a subset of codons with signatures of positive selection using codon-based likelihood analysis based on the observed $d_{\mathrm{N}} / d_{\mathrm{S}}$ rate ratio (Yang and Bielawski 2000). The parameters defining the codon evolution model used in this study were derived by capturing heterogeneity of $d_{\mathrm{N}} / d_{\mathrm{S}}$ across multiple sites, with positive selection inferred when $d_{\mathrm{N}} / d_{\mathrm{S}}>1$. Under the codon model that provided the best fit to the data, model M2, about $10 \%$ of codons showed potential evidence of positive selection (average $d_{\mathrm{N}} / d_{\mathrm{S}}=2.95$ ). However, despite the demonstration of evolutionary patterns consistent with the effects of positive selection, none of the amino acid sites in gap-free alignment columns in the $p 67$ gene identified by maximum likelihood analysis had a highly significant ( $>95 \%)$ posterior probability of being positively selected (Table 4). It is possible that the high $d_{\mathrm{N}} / d_{\mathrm{S}}$ ratio observed for several amino acids results from allelic dynamics and does not accurately reflect the effect of selection (Kryazhimskiy and Plotkin 2008). Interestingly, none of the codons located within defined linear epitopes recognized by monoclonal antibodies shows signatures of positive selection. These results are consistent with a recent study that showed no preferential distribution of sites under positive selective pressure within $\mathrm{T}$ cell epitopes in candidate vaccine antigens from the pathogenic T. parva schizonts (Pelle et al. 2011). Among the 115 cattle exposed to challenge at Marula, from which the 18 isolates analyzed were derived, approximately $80 \%$ of those that developed severe disease and died exhibited features associated with corridor disease, specifically low schizont parasitosis and piroplasm parasitemia, irrespective of whether they were isolated from vaccinated or control cattle (R. Bishop and A. Musoke, unpublished data). One conclusion from this study is therefore that T. parva parasites originating from buffalo can infect and cause severe disease in co-grazing cattle and have diverse p67 genotypes, unlike the conserved p67 sequence observed in T. parva populations that have adapted to transmission between cattle. The data also suggests that buffaloderived T. parva can sometimes "break through" the immunity induced by ITM immunization and induce severe clinical reactions, in areas with a high tick challenge.

Acknowledgments We are grateful to Dr. Jan Naessens for proofreading the manuscript. The work was supported by funding from the DFG German-African Cooperation Projects in Infectiology: "Molecular epidemiology network for promotion and support of delivery of life vaccines against Theileria parva and Theileria annulata infection in Eastern and Northern Africa (SE 862/2-1)." This is ILRI publication number 92-9146-369-8.

Open Access This article is distributed under the terms of the Creative Commons Attribution License which permits any use, distribution, and reproduction in any medium, provided the original author(s) and the source are credited.

\section{References}

Altschul SF, Gish W, Miller W, Myers EW, Lipman DJ (1990) Basic local alignment search tool. J Mol Biol 215:403-410

Bishop R, Nene V, Staeyert J, Rowlands J, Nyanjui J, Osaso J, Morzaria S, Musoke A (2003) Immunity to East Coast fever in cattle induced by a polypeptide fragment of the major surface coat protein of Theileria parva sporozoites. Vaccine 21:1205-1212

Blackman MJ, Scott-Finnigan TJ, Shai S, Holder AA (1994) Antibodies inhibit the protease-mediated processing of a malaria merozoite surface protein. J Exp Med 13:389-393

Dahlbäck M, Rask TS, Andersen PH, Nielsen MA, Ndam NT, Resende M, Turner L, Deloron P, Hviid L, Lund O, Pedersen AG, Theander TG, Salanti A (2006) Epitope mapping and topographic analysis of VAR2CSA DBL3X involved in P. falciparum placental sequestration. PLoS Pathog 2(11):e124

Gouveia-Oliveira R, Sackett PW, Pedersen AG (2007) MaxAlign: maximizing usable data in an alignment. BMC Bioinforma 8:312

Katoh K, Misawa K, Kuma K, Miyata T (2002) MAFFT: a novel method for rapid multiple sequence alignment based on fast Fourier transform. Nucleic Acids Res 30:3059-3066

Kryazhimskiy S, Plotkin JB (2008) The population genetics of dN/dS. PLoS Genet 4(12):e1000304

Librado P, Rozas J (2009) DnaSP v5: a software for comprehensive analysis of DNA polymorphism data. Bioinformatics 25:1451-1452

McKeever DJ, Taracha EL, Innes EL, MacHugh ND, Awino E, Goddeeris BM, Morrison WI (1994) Adoptive transfer of immunity to Theileria parva in the $\mathrm{CD} 8^{+}$fraction of responding efferent lymph. Proc Natl Acad Sci U S A 91:1959-1963

McKeever DJ, Taracha EL, Morrison WI, Musoke AJ, Morzaria SP (1999) Protective immune mechanisms against Theileria parva: evolution of vaccine development strategies. Parasitol Today 15: 263-267

Morrison WI (2009) Progress towards understanding the immunobiology of Theileria parasites. Parasitology 136:1415-1426

Morzaria SP, Dolan TT, Norval RA, Bishop RP, Spooner PR (1995) Generation and characterisation of cloned Theileria parva parasites. Parasitology 111:39-49

Morzaria S, Spooner P, Bishop R, Mwaura S (1999) The preparation of a composite stabilate for immunisation against East Coast fever. In: Morzaria S, Williamson S (eds) Live vaccines for Theileria parva: deployment in eastern, central and southern Africa. International Livestock Research Institute, Kenya, pp 56-61

Musoke A, Morzaria S, Nkonge C, Jones E, Nene V (1992) A recombinant sporozoite surface antigen of Theileria parva induces protection in cattle. Proc Natl Acad Sci U S A 89:514-518

Musoke A, Rowlands J, Nene V, Nyanjui J, Katende J, Spooner P, Mwaura S, Odongo D, Nkonge C, Mbogo S, Bishop R, Morzaria S (2005) Subunit vaccine based on the p67 major surface protein of Theileria parva sporozoites reduces severity of infection derived from field tick challenge. Vaccine 23:3084-3095

Nene V, Musoke A, Gobright E, Morzaria S (1996) Conservation of the sporozoite p67 vaccine antigen in cattle-derived Theileria parva 
stocks with different cross-immunity profiles. Infect Immun 64: 2056-2061

Nene V, Gobright E, Bishop R, Morzaria S, Musoke A (1999) Linear peptide specificity of bovine antibody responses to p67 of Theileria parva and sequence diversity of sporozoite-neutralizing epitopes: implications for a vaccine. Infect Immun 67:1261-1266

Norval RAI, Perry BD, Young AS (1992) The epidemiology of Theileria in Africa. Academic, London

Oura CA, Tait A, Asiimwe B, Lubega GW, Weir W (2011) Theileria parva genetic diversity and haemoparasite prevalence in cattle and wildlife in and around Lake Mburo National Park in Uganda. Parasitol Res 108:1365-1374

Payne R (1999) Preparation of stabilates for immunisation against East Coast fever at NVRC, Muguga, Kenya. In: Morzaria S, Williamson $\mathrm{S}$ (eds) Live vaccines for Theileria parva: deployment in eastern, central and southern Africa. International Livestock Research Institute, Kenya, pp 62-65

Pelle R, Graham SP, Njahira MN, Osaso J, Saya RM, Odongo DO, Toye PG, Spooner PR, Musoke AJ, Mwangi DM, Taracha EL, Morrison WI, Weir W, Silva JC, Bishop RP (2011) Two Theileria parva CD8 $T$ cell antigen genes are more variable in buffalo than cattle parasites, but differ in pattern of sequence diversity. PLoS One 6(4):e19015

Posada D, Buckley TR (2004) Model selection and model averaging in phylogenetics: advantages of Akaike information criterion and Bayesian approaches over likelihood ratio tests. Syst Biol 53: 793-808

Posada D, Crandall KA (1998) MODELTEST: testing the model of DNA substitution. Bioinformatics 14:817-818

Radley DE, Brown CGD, Burridge MJ, Cunningham MP, Kirimi IM, Purnell RE, Young AS (1975) Chemoprophylactic immunisation of cattle against Theileria parva (Muguga) and five Theileria strains. Vet Parasitol 1:35-41

Saitou N, Nei M (1987) The neighbor-joining method: a new method for reconstructing phylogenetic trees. Mol Biol Evol 4:406-425

Sibeko KP, Geysen D, Oosthuizen MC, Matthee CA, Troskie M, Potgieter FT, Coetzer JA, Collins NE (2010) Four p67 alleles identified in South African Theileria parva field samples. Vet Parasitol 167:244-254

Swofford DL (2002) PAUP: phylogenetic analysis using parsimony and other methods. Version 4. Sinauer Associates, Sunderland

Taracha EL, Goddeeris BM, Teale AJ, Kemp SJ, Morrison WI (1995) Parasite strain specificity of bovine cytotoxic $\mathrm{T}$ cell responses to Theileria parva is determined primarily by immunodominance. $\mathrm{J}$ Immunol 155:4854-4860

Tetteh KK, Cavanagh DR, Corran P, Musonda R, Mcbride JS, Conway DJ (2005) Extensive antigenic polymorphism within the repeat sequence of the Plasmodium falciparum merozoite surface protein 1 block 2 is incorporated in a minimal polyvalent Immunogen. Infect Immun 73:5928-5935

Thomsen MC, Nielsen M (2012) Seq2Logo: a method for construction and visualization of amino acid binding motifs and sequence profiles including sequence weighting, pseudo counts and two-sided representation of amino acid enrichment and depletion. Nucleic Acids Res 40:W281-W287

Wagenmakers EJ, Farrell S (2004) AIC model selection using Akaike weights. Psychon Bull Rev 11:192-196

Wallach MG, Ashash U, Michael A, Smith NC (2008) Field application of a subunit vaccine against an enteric protozoan disease. PLoS One 3:e3948

Weedall GD, Preston BM, Thomas AW, Sutherland CJ, Conway DJ (2007) Differential evidence of natural selection on two leading sporozoite stage malaria vaccine candidate antigens. Int J Parasitol 37:77-85

Yang Z (2007) PAML 4: phylogenetic analysis by maximum likelihood. Mol Biol Evol 24:1586-1591

Yang Z, Bielawski JP (2000) Statistical methods for detecting molecular adaptation. Trends Ecol Evol 15:496-503

Yang Z, Goldman N, Friday A (1994) Comparison of models for nucleotide substitution used in maximum-likelihood phylogenetic estimation. Mol Biol Evol 11:316-324

Yang Z, Wong WS, Nielsen R (2005) Bayes empirical Bayes inference of amino acid sites under positive selection. Mol Biol Evol 22: $1107-1118$ 